\title{
Induction of Tumor Growth After Preoperative Portal Vein Embolization: Is It a Real Problem?
}

\author{
Wilmar de Graaf ${ }^{1}$, Jacomina W. van den Esschert ${ }^{1}$, Krijn P. van Lienden ${ }^{2}$, and Thomas M. van Gulik ${ }^{1}$ \\ ${ }^{1}$ Department of Surgery, Academic Medical Center, Meibergdreef 9, IWO-1, Amsterdam 1105, AZ, The Netherlands; \\ ${ }^{2}$ Department of Radiology, Academic Medical Center, Amsterdam, The Netherlands
}

\begin{abstract}
Although preoperative portal vein embolization (PVE) is an effective means to increase future remnant liver (FRL) volume, little has been published on possible adverse effects. This review discusses the clinical and experimental evidence regarding the effect of PVE on tumor growth in both embolized and nonembolized liver lobes, as well as potential strategies to control tumor progression after PVE. A literature review was performed using MEDLINE with keywords related to experimental and clinical studies concerning PVE, portal vein ligation (PVL), and tumor growth. Cross-references and references from reviews were also checked. Clinical and experimental data suggest that tumor progression can occur after preoperative PVE in embolized and nonembolized liver segments. Clinical evidence indicating possible tumor progression in patients with colorectal metastases or with primary liver tumors is based on studies with small sample size. Although multiple studies demonstrated tumor progression, evidence concerning a direct increase in tumor growth rate as a result of PVE is circumstantial. Three possible mechanisms influencing tumor growth after PVE can be recognized, namely changes in cytokines or growth factors, alteration in hepatic blood supply and an enhanced cellular host response promoting local tumor growth after PVE. Post-PVE chemotherapy and sequential transcatheter arterial chemoembolization (TACE) before PVE have been proposed to reduce tumor mass after PVE. We conclude that tumor progression can occur after PVE in patients with colorectal metastases as well as in patients with primary liver tumors. However, further research is needed in order to rate this risk of tumor progression after PVE.
\end{abstract}

(C) The Author(s) 2008. This article is published with open access at Springerlink.com

First Received: 22 July 2008;

Published Online: 3 December 2008

T. M. van Gulik

e-mail: T.m.vangulik@amc.uva.nl
Preoperative portal vein embolization (PVE) is an accepted intervention in patients requiring major liver resection in whom the estimated future remnant liver (FRL) is too small to allow safe resection. ${ }^{1,2}$ PVE induces atrophy of the embolized, tumor-bearing liver lobe while compensatory hypertrophy of the nonembolized lobe occurs, thereby increasing FRL volume and function. ${ }^{1-6}$ Portal vein occlusion by either embolization (PVE) or ligation (PVL) has proven useful to reduce risk of postoperative liver dysfunction and enables resection in patients previously deemed unresectable due to a marginal FRL. ${ }^{7-14}$ FRL volume smaller than $25-30 \%$ of total preoperative liver volume is generally considered insufficient in patients with normal liver parenchyma. ${ }^{15-17}$ In patients with a compromised liver, the cutoff value for performing safe resection is more variable and PVE is usually undertaken when the FRL is smaller than $40 \%$ of total liver volume. ${ }^{10,12,16}$

While experiences with PVE have accumulated, there is growing evidence that PVE stimulates not only the growth of the FRL but also affects tumor size in both embolized and nonembolized liver segments. ${ }^{18-20}$ This review discusses the clinical and experimental evidence regarding the effect of PVE on tumor growth in the nonembolized and embolized liver lobes, as well as potential strategies to control tumor progression after PVE.

\section{THE EFFECT OF PVE ON TUMOR GROWTH}

Table 1 provides an overview of the studies describing tumor progression after preoperative PVE. Elias et al. were among the first to describe the potential of intrahepatic tumor enlargement after PVE. ${ }^{18}$ Their conclusion was based on five patients with known tumors in the nonembolized liver lobes. All patients had measurable tumors in the left (nonembolized) liver lobes before PVE, which provided the opportunity to measure tumor enlargement. In four of the five patients tumor size increased after PVE. 
TABLE 1 Studies describing the effect of preoperative PVE on tumor progression

\begin{tabular}{|c|c|c|c|c|c|}
\hline & Tumor type & Tumor location & $\begin{array}{l}\text { No. of } \\
\text { patients }\end{array}$ & Increase in tumor volume $(\%)$ & $\begin{array}{l}\text { Increase in } \\
\text { growth rate }(\%)\end{array}$ \\
\hline Elias et al. ${ }^{18}$ & Liver metastases & Nonembolized liver segments & 5 & $215 \%(-30 \%, 970 \%)^{\mathrm{a}}$ & - \\
\hline \multirow[t]{2}{*}{ Hayashi et al. ${ }^{24}$} & $\mathrm{HCC}$ & Embolized liver segments & 6 & - & $265 \%(200-746 \%)^{\mathrm{a}}$ \\
\hline & $\mathrm{CCC}$ & Embolized liver segments & 2 & - & $116 \%(100-132 \%)^{\mathrm{a}}$ \\
\hline \multirow[t]{2}{*}{ Kokudo et al. $^{20}$} & CRM & Embolized liver segments & 15 & $20.8 \%^{\mathrm{b}}$ & - \\
\hline & & $\begin{array}{l}\text { Both embolized and } \\
\text { nonembolized }\end{array}$ & 3 & $\begin{array}{l}3.0 \%(2.5-6.3 \%)^{\mathrm{a}} \text { (embolized) } \\
9.7 \%(0.5-42.1 \%)^{\mathrm{a}} \text { (nonembolized) }\end{array}$ & - \\
\hline \multirow[t]{2}{*}{ Barbaro et al. ${ }^{19}$} & CRM & Embolized liver segments & 6 & $84.4 \%(62.4-562 \%)^{\mathrm{a}}$ & - \\
\hline & Carcinoid tumor & Embolized liver segments & 3 & 0 & - \\
\hline
\end{tabular}

$C R M$ colorectal metastasis, $H C C$ hepatocellular carcinoma, $C C C$ cholangiocarcinoma

${ }^{\text {a }}$ Median (minimum, maximum)

b Mean

One patient with impaired liver function showed no increase in tumor volume. The authors concluded that, in patients with normal liver parenchyma, the growth rate of metastases is more rapid than the hypertrophy of the surrounding liver parenchyma. Although the increase in tumor size in this study was impressive, no tumor growth rate before PVE was investigated, making it impossible to draw conclusions regarding direct stimulation of tumor growth by PVE. The study only demonstrates that tumor size can increase in the waiting period between PVE and resection. An increased growth rate of liver metastases as a result of portal vein diversion was however demonstrated in a murine model in which the portal vein to one side of the liver was ligated. ${ }^{21}$ Tumor increase in the nonembolized segments is of special interest in patients with bilateral liver metastases. Although in these patients a right hemihepatectomy with simultaneous wedge resection in the left liver has been described after right PVE, the outgrowth of lesions in the left liver in the time between right PVE and resection poses a potential threat in respect with resectability. ${ }^{22}$ A two-staged resection in combination with PVE can successfully be applied in some of these patients. ${ }^{23}$ In the first stage resection removal of all tumor mass located in the (future) nonembolized liver segments is required before the actual PVE (or PVL) in order to prevent rapid tumor enlargement after portal vein occlusion. ${ }^{23}$ Subsequently, major hepatectomy can be performed several weeks after the portal vein occlusion.

The time between PVE and follow-up computed tomography (CT) to recalculate FRL volume can also be viewed as a diagnostic window in which clinical outgrowth of micrometastases in the nonembolized lobe may occur. Small metastases in the nonembolized lobe, not detectable on a CT scan before embolization, may become visible after PVE as a result of the potential tumor-growth-stimulating environment provided by PVE. Such a finding will render the patient unresectable or will require reconsideration of management (Fig. 1).

Several studies have described the effect of preoperative PVE on progression of colorectal metastases in the embolized liver segments. Kokudo et al. were the first to report increased proliferative activity of colorectal liver metastases. $^{20}$ This is the largest case series concerning this issue, including 18 patients with prior PVE who were
FIG. 1 Example of a patient with multiple colorectal metastases in the right liver lobe requiring an extended right hemihepatectomy. No lesions in segment 2 and 3 were visible (a). Due to a small FRL volume, PVE was performed. CT scan 3 weeks after PVE revealed multiple metastases in segments 2 and 3 excluding this patient from resection (b)
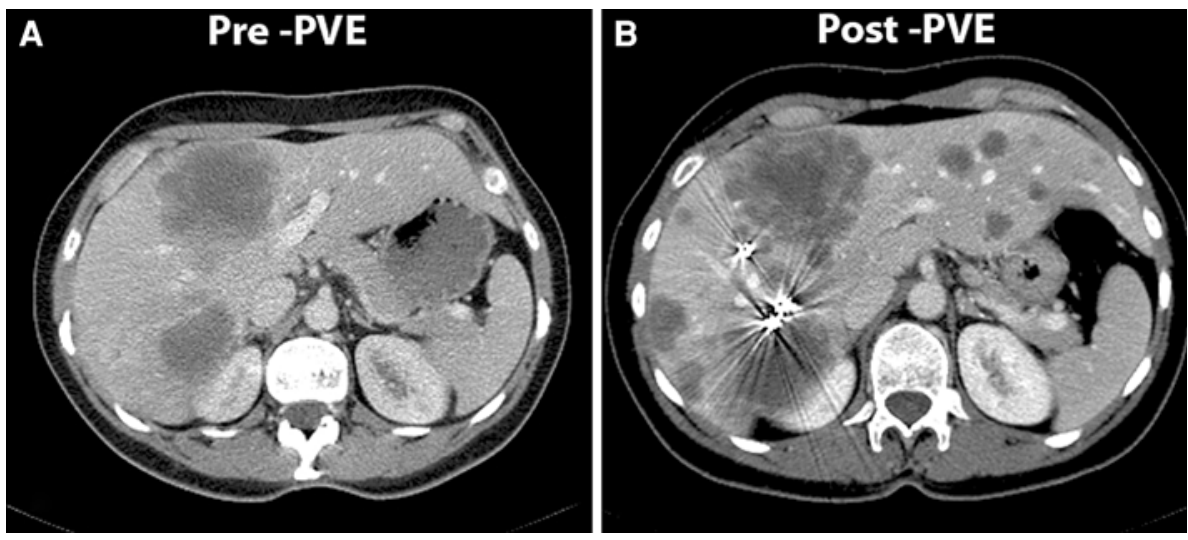
compared with 29 patients who were resected without PVE. One patient in the PVE group received preoperative intra-arterial chemotherapy. No use of neoadjuvant chemotherapy was described in the other patients. Mean tumor volume, measured by CT volumetry, was significantly increased by $20.8 \%$ in the 3 -week interval after PVE. However, the growth of liver tumors in the group without PVE was not assessed, and tumor growth rate before PVE was not measured. Instead, the proliferation of metastatic lesions measured by Ki-67 labeling index after PVE was compared with the proliferation rate of metastatic lesions in the control group undergoing only resection. Ki-67 labeling index was significantly higher in the PVE group. Although the distribution of well, moderately, and poorly differentiated adenocarcinoma was similar within the two groups, tumor size was significantly larger in the PVE group, which influences the Ki-67 labeling index. This study only demonstrates that colorectal liver metastases continue to grow in the embolized liver lobes after PVE. The evidence of direct tumor growth stimulation by PVE is however circumstantial. Barbaro et al. confirmed the increase of tumor volume after PVE in patients with colorectal metastases. ${ }^{19}$ In contrast, no increase in tumor volume was observed in patients with carcinoid metastases, suggesting that tumor characteristics are important for tumor progression after PVE. Again no evidence is provided in this study to demonstrate direct stimulation of tumor growth by PVE. Figure 2 demonstrates tumor enlargement in the embolized segments after PVE in a patient with colorectal metastases.

Data also exist that the growth of primary liver tumors including hepatocellular carcinoma (HCC) and cholangiocellular carcinoma (CCC) is influenced by PVE. ${ }^{24}$ In eight patients with primary liver tumors (six HCC, two CCC), rate of tumor growth after PVE was compared with growth rate of the same tumors in a period before embolization. Tumor growth rate accelerated from $0.59 \mathrm{~cm}^{3} /$ day before PVE to $2.37 \mathrm{~cm}^{3} /$ day after PVE, as measured by CT volumetry. This is the first study demonstrating induction of increased tumor growth by PVE as compared with the growth rate before PVE in the same patients.

In a recent study, Ribero et al. retrospectively analyzed 112 PVE patients. ${ }^{13}$ Changes in tumor size were measured in 80 patients, resulting in overall no increase in median tumor size within all patients after PVE. The change in 95\% confidence interval after PVE indicates both an increase and a decrease in some patients. However, no information was provided regarding the percentage of patients with increased or decreased tumor size after PVE. Twenty-eight patients received chemotherapy prior to PVE and five patients received chemotherapy 2 weeks after PVE. No differentiation was made between patients with or without chemotherapy, and no tumor volumes were calculated. Additional studies are therefore required to precisely assess the risk of accelerated tumor growth in patients receiving PVE.

Besides reports of tumor progression after PVE, studies describe high percentages of patients with unresectable disease after PVE. In the study by Ribero et al. 10 out of the 112 patients (9\%) did not undergo surgery after PVE because of extra- or intrahepatic disease progression. ${ }^{13}$ An additional 12 patients (11\%) had unexpected extra- or intrahepatic disease that became evident at laparotomy. Similar percentages of tumor progression precluding curative resection are described, with percentages ranging from $6.4 \%$ to $33 \% .^{8,11,25,26}$ A recent meta-analysis assessing the results of preoperative PVE reported that $85 \%$ of the evaluable patients that had undergone PVE underwent laparotomy. Of these patients, $11.3 \%$ were unresectable due to intra- or extrahepatic tumor spread. ${ }^{1}$

The effect of tumor progression on disease-free and overall survival is currently elusive. In the study by Kokudo et al. disease-free survival at 2 and 4 years in patients who had undergone PVE was significantly poorer than in those undergoing partial liver resection without prior PVE. However, the two groups were different with respect to preoperative tumor diameter and use of postoperative
FIG. 2 Example of a patient with a large colorectal metastasis in the right liver. CT scan 3 weeks after PVE shows a clear increase in tumor diameter. Total tumor volume increased by $42 \%$
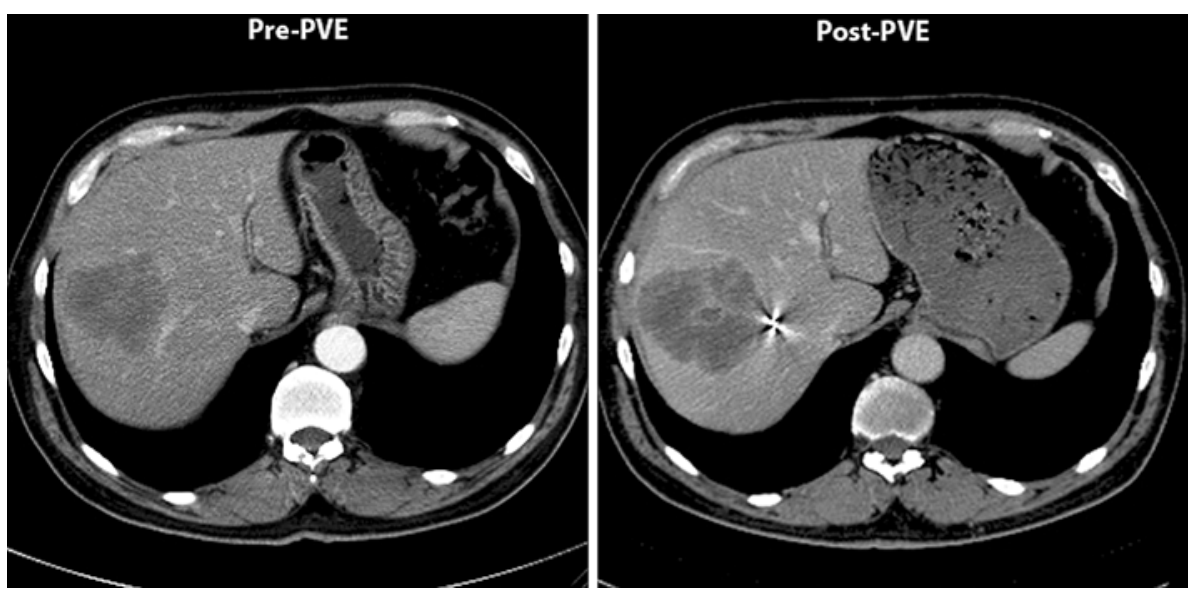
chemotherapy, making direct comparison impossible. In addition, no difference in overall survival was found. Azoulay et al. demonstrated similar survival rates after hepatectomy with or without previous PVE. Only resected patients were included in the survival analysis, and $33 \%$ of the patients with previous PVE were unresectable due to tumor progression. Survival rate in these patients was significantly poorer.

Little evidence exists regarding the effect of PVE on the induction of distant metastases. Breakdown and remodeling of the extracellular matrix takes place especially in the embolized, tumor-bearing liver lobes. Matrix metalloproteinases (MMPs) are most likely involved in this remodeling process similar to remodeling during liver regeneration. $^{27}$ MMPs have been reported to promote metastatic behavior in several types of tumors, including colorectal cancer. $^{28}$

\section{MECHANISMS AFFECTING TUMOR GROWTH AFTER PVE}

Three possible mechanisms inducing tumor growth after PVE have been proposed, namely changes in cytokines and growth factors, alteration in hepatic blood supply, and enhanced cellular host response promoting local tumor growth.

\section{Cytokines and Growth Factors}

The mechanisms underlying the atrophy-hypertrophy complex induced after PVE remain largely undetermined. Growth factors such as interleukin (IL)-6, tumor necrosis factor (TNF)- $\alpha$, and hepatocyte growth factor (HGF) are upregulated and play an essential role during liver regeneration after partial liver resection. ${ }^{29}$ The same growth factors have been implicated in stimulating growth of colorectal carcinoma cells in vitro. ${ }^{30,31}$ The HGF receptor is present in almost all human colorectal carcinomas. ${ }^{32}$ Treatment of human colon carcinoma cell lines with HGF stimulates cell growth and increases its metastatic potential. $^{32,33}$ Experimental studies have shown an increase in IL-6, TNF- $\alpha$, and HGF mRNA expression in the nonligated, hypertrophied liver lobes after PVL, suggesting a similar role of these factors as in post-hepatectomy liver regeneration. ${ }^{20,34-36}$ Local elevation of these growth factors may stimulate colorectal metastases in the nonembolized liver segments. Interestingly, it has also been demonstrated that HGF and IL-6, although to a lesser extent, are upregulated in the ipsilateral liver lobes after PVL. $^{20,35,36}$ In addition, increased tissue levels of HGF may increase plasma levels, thus stimulating the growth of hepatic tumors in the embolized lobe.
A recent experimental study demonstrated that PVL induced sinusoidal perfusion failure along with significant hypoxia during the initial few days after PVL, resulting in necrosis and apoptosis in the ligated liver tissue. ${ }^{37} \mathrm{How}-$ ever, most malignant tumors tolerate hypoxia quite well. ${ }^{38}$ In addition, hypoxia may induce cellular changes that can result in more aggressive phenotypes with increased potential for local invasive growth, distant tumor spreading, and resistance to therapy. ${ }^{38,39}$ In the first days after PVL negative regulators of hepatocyte proliferation, such as transforming growth factor- $\beta$ (TGF- $\beta$ ) and interleukin-1 (IL-1), are strongly expressed in the atrophic lobes. ${ }^{36}$ TGF$\beta$ serves in normal tissue as a tumor suppressor by inhibiting cell proliferation. Many colorectal carcinomas, however, are resistant to TGF- $\beta$-induced growth inhibition. In advanced stages, TGF- $\beta$ can even stimulate the proliferation of colon carcinoma cells. ${ }^{40}$ In the late phase after ligation, tissue remodeling takes place which is dominated by cell proliferation. ${ }^{37}$ Hence, tumor proliferation may be promoted in this late phase after PVE.

\section{Alteration in Hepatic Blood Supply}

Increased hepatic arterial blood flow after embolization of the ipsilateral portal branch is another factor potentially stimulating tumor growth after PVE. The liver has a dual blood supply with about $75 \%$ being contributed by the portal vein and $25 \%$ by the hepatic artery. ${ }^{41}$ Compensatory increased arterial perfusion, known as the hepatic arterial buffer response, occurs after reduction of segmental portal blood flow. ${ }^{42}$ Clinical and experimental studies demonstrate a significant increase in hepatic arterial blood flow in the occluded liver lobes resulting from an increase in common hepatic arterial flow. ${ }^{37,43}$ After portal vein occlusion, sinusoidal perfusion is derived almost totally from arterial blood supply. ${ }^{44}$ Because liver tumors are mainly fed by the hepatic artery, the hepatic arterial buffer response potentially stimulates tumor growth in the embolized liver lobes.

Although a very likely theory, no studies as yet have proven the involvement of the hepatic arterial buffer response in the induction of tumor growth

\section{Cellular Host Response Promoting Local Tumor Growth}

A third mechanism implicated in tumor growth after PVE is the local cellular response evoked in the embolized atrophying liver lobes. ${ }^{45}$ Studies in animal models have demonstrated that portal vein occlusion induces an immediate early gene response in both ligated and nonligated liver lobes. ${ }^{35,45}$ Enhanced expression of several of these genes in the atrophying liver tissue such as heat shock 
protein-70 (hsp70), heme oxygenase-1 (hmox-1), or plasminogen activator inhibitors $(P A I-1)$ have a cytoprotective effect and a role in tissue remodeling and repair. ${ }^{45}$ The same factors have been shown to facilitate growth and angiogenesis in solid tumors, including colon carcinomas. ${ }^{46-49}$ The production of these factors by the surrounding liver parenchyma may in this manner contribute to tumor progression. In addition, many tumors including HCC and colorectal carcinomas have the potential to express $h s p 70$ themselves. ${ }^{50}$ In some tumors, expression of $h s p 70$ has been related to cell proliferation, poor prognosis, and resistance to chemotherapy. ${ }^{50}$ Similar to the local parenchyma, tumor cells might upregulate hsp70 in response to occlusion of portal blood flow, thereby increasing their proliferative capacity.

\section{STRATEGIES TO REDUCE TUMOR GROWTH AFTER PVE}

Several therapeutic strategies have been described to prevent and even reduce tumor progression after PVE including sequential hepatic transcatheter arterial chemoembolization (TACE) and PVE and post-PVE chemotherapy.

\section{Sequential TACE and PVE}

Studies from Japan reported that additional ipsilateral TACE before or after PVE improved the hypertrophy response of the FRL in patients with HCC and chronic liver disease ${ }^{51,52}$ The rationale behind this combination was not only to improve the regenerative capacity after PVE by closing down arterial-portal shunts but also to reduce the risk of tumor progression secondary to the compensatory increase in arterial blood flow. ${ }^{51,53-55}$ Aoki et al. described a group of $17 \mathrm{HCC}$ patients who underwent PVE 710 days after TACE. ${ }^{51}$ The combination generated sufficient hypertrophy of the nonembolized lobes within 2 weeks. No tumor progression was noted in the waiting time until resection as measured by CT volumetry. Examination of the resection specimen revealed almost complete tumor necrosis $(90-100 \%)$ in ten patients $(59 \%)$. In the other patients, extent of necrosis was $50-80 \%$. Ogata et al. compared a group of HCC patients $(n=18)$ undergoing PVE 3 weeks after TACE with a group of HCC patients who underwent only right-sided PVE in the same period. ${ }^{54}$ Right hemihepatectomy was performed 48 weeks after PVE. Mean increase in FRL volume and rate of hypertrophy were significantly higher in the group in which PVE was combined with TACE. Using this combination, complete tumor necrosis was achieved in $80 \%$ of the patients compared with $5 \%$ in the PVE group. In addition 1-, 3-, and 5-year recurrence-free survival rates were higher in the combined group. This clearly demonstrates that TACE combined with PVE is effective in reducing tumor progression in $\mathrm{HCC}$ patients.

A drawback of the combination of TACE with PVE is the risk of ischemic parenchymal damage. Vetelainen et al. demonstrated that simultaneous ligation of the hepatic artery and portal vein in rats resulted in massive liver cell necrosis with increased systemic inflammatory response and decreased liver function. ${ }^{56}$ An interval of $48 \mathrm{~h}$ between both procedures decreased the risk of liver injury. Nakao et al. reported, in a clinical study, that simultaneous hepatic arterial and portal venous embolization resulted in necrosis and infarction of the embolized tissue. ${ }^{53}$ Aoki et al. used intervals of 7-10 days in their clinical study and described a transient increase in liver damage parameters. ${ }^{51}$ Examination of the resection specimen showed minimal necrosis of the liver parenchyma in the majority of patients. Two patients however had segmental infarction in the embolized lobes. Ogata et al. used time intervals of 3-4 weeks which resulted in significant increase in aspartate aminotransferase (AST)/alanine aminotransferase (ALT) levels, however without decrease in liver function. ${ }^{54}$ The most suitable interval between ipsilateral arterial and portal embolization in the clinical setting remains uncertain as well as its effect in livers compromised by steatosis or previous chemotherapy.

Although TACE is most frequently used in patients with hepatocellular carcinoma, there are also reports indicating its use in other liver tumors including colorectal metastases. ${ }^{57-59}$ This suggests that sequential TACE with preoperative PVE could also be potentially beneficial in patients with colorectal metastases. To date, however, no study has reported the using of sequential TACE with preoperative PVE in this category of patients.

\section{Post-PVE Chemotherapy}

With recent development of improved chemotherapeutic agents, an increasing number of patients with metastatic liver disease are treated with neoadjuvant chemotherapy. If preoperative PVE is required, chemotherapy is discontinued several weeks prior to embolization until surgery 34 weeks thereafter, because of its alleged negative influence on the hypertrophy response. ${ }^{11,60,61}$ This allows tumor progression to occur in the period in which chemotherapy is discontinued in addition to the possible tumor stimulating effects of PVE. Beal et al. reported in a retrospective study including 15 patients, of which 10 received post-PVE chemotherapy, that hypertrophy of the FRL did occur in the post-PVE chemotherapy group, although significantly less compared with the nonchemotherapy group. ${ }^{60}$ Tumor progression was seen in four of the five patients without post-PVE chemotherapy, whereas tumor reduction was seen in six of the ten patients with chemotherapy. 
The group of Belghiti recently demonstrated no significant difference in hypertrophy response nor in postoperative complications when chemotherapy was continued after PVE. ${ }^{61}$ They therefore recommended not to disrupt a successful chemotherapy course prior to or after portal vein occlusion in patients with colorectal metastases. These conclusions however, are based on a series of 20 patients, of whom only 10 received post-PVE chemotherapy.

Covey et al. recently confirmed that continuation of chemotherapy after PVE had no negative influence on the hypertrophy response in a series of 100 patients including 43 patients with post-PVE chemotherapy. ${ }^{62}$ Interestingly, significantly more patients were unresectable in the postPVE chemotherapy group. The reasons for unresectability of each group were not given but, overall, $23 \%$ of the patients were not resected due to intra- and/or extrahepatic tumor progression. This difference can be caused by a selection bias between the two groups because tumor size and stage were also not provided.

Selzner et al. applied selective intrahepatic arterial (SIHA) chemotherapy combined with portal vein ligation for downstaging of colorectal liver metastases. ${ }^{63}$ SIHA chemotherapy has the advantage of a high response rate with a low rate of systemic toxicity. SIHA was started 37 days after PVL in 11 patients using a catheter positioned in the gastroduodenal artery with the tip at the junction with the hepatic artery. Chemotherapy involved serial administration of floxuridine for 2 weeks every 4 weeks. The volume of liver metastases decreased by $60 \%$ within 3 months after PVL. Although this study was performed in a highly selected group of unresectable patients, it shows that chemotherapy is able to reduce tumor growth after ipsilateral portal vein occlusion.

\section{DISCUSSION}

PVE as a means to induce hypertrophy of the FRL is clearly established. Little has been reported, however, about the negative side-effects of PVE. Several studies have shown tumor progression in patients with colorectal metastases as well as in patients with primary liver tumors after PVE. ${ }^{18-20,24}$ Although the clinical studies clearly demonstrate that tumor progression is possible in both the embolized and nonembolized liver lobes, the evidence of direct stimulation of tumor growth by PVE is circumstantial. In addition the reports of increased tumor proliferation after PVE are based on limited case series. In a larger study, no increase in median tumor size after PVE was shown within the total group of 80 patients. ${ }^{13}$ However, to date, there is no information available on the percentage of patients with increased or decreased tumor size after PVE. Additional studies are therefore required to more precisely assess the risk of accelerated tumor growth in patients receiving PVE.
Tumor progression after PVE creates a dilemma in terms of optimal waiting time until resection. The risk of tumor growth obviously demands as short as possible waiting time. The time interval is mainly determined by the time required to attain sufficient FRL volume. Usually a period of 3-4 weeks is considered sufficient based on CT volumetry. ${ }^{64}$ Little is known concerning the improvement of FRL function after PVE. One study separately assessed biliary excretion of indocyanine green by the embolized and nonembolized liver lobes and concluded that the functional gain in the nonembolized lobes was of greater magnitude than the volumetric increase. ${ }^{6}$ Two additional studies from Japan confirmed that the increase in FRL function after PVE measured by technetium-99 $\mathrm{m}\left({ }^{99 \mathrm{~m}} \mathrm{Tc}\right)$ galactosyl-human serum albumin (GSA) scintigraphy exceeds the increase in volume in cirrhotic and noncirrhotic patients. ${ }^{3,65}$ This implies that the recommended waiting time until operation may be shorter than suggested by volumetric studies, which is more favorable in light of the risk of tumor progression after PVE.

The combination of TACE before PVE is effective in inducing tumor necrosis and thereby in inhibiting tumor progression after PVE. ${ }^{51,53,54}$ There is nevertheless, a risk of massive necrosis with serious complications. The time interval between the two procedures is therefore crucial to safely undertake the combination. More research is needed to define which patients benefit most from sequential TACE and PVE and to determine the optimal time interval between both procedures.

Neoadjuvant chemotherapy has become increasingly important in downsizing unresectable colorectal liver metastases and there are also data indicating its use in initially resectable cases. ${ }^{66}$ Continuation of chemotherapy after PVE is a concern as, during that time, regeneration of FRL has to take place. Since the inhibitory effect of chemotherapy on the hypertrophy response appears to be less than previously assumed, there seems to be a place for chemotherapy after PVE to control tumor growth in patients with colorectal metastases, particularly in those who previously have shown to be good responders. ${ }^{60,61}$ Systemic chemotherapy may also have the advantage of controlling the progression of extrahepatic disease in the waiting time until resection. This is an area for controlled studies to further determine the role of chemotherapy after PVE.

The high percentage of patients reported to have unresectable disease after PVE, allegedly due to tumor progression, is another issue. No studies are available concerning the follow-up of unresectable patients after PVE. The described effect of PVE stimulating tumor growth raises the question of whether PVE reduces survival in comparison with unresectable patients who did not undergo PVE. 
In conclusion, whereas PVE is an established method to increase the rate of patients with resectable liver tumors, several issues need to be further clarified. PVE allows tumor progression in both the embolized and nonembolized liver segments. Although clinical studies clearly demonstrate that tumor progression after PVE is possible, accurate data regarding the risk of tumor progression after PVE are currently not available. However, the possibility of tumor progression makes it important to minimize unnecessary waiting time between PVE and resection. Sequential TACE with PVE as well as post-PVE chemotherapy are promising strategies to control tumor progression after PVE.

OPEN ACCESS This article is distributed under the terms of the Creative Commons Attribution Noncommercial License which permits any noncommercial use, distribution, and reproduction in any medium, provided the original author(s) and source are credited.

\section{REFERENCES}

1. Abulkhir A, Limongelli P, Healey AJ, et al. Preoperative portal vein embolization for major liver resection: a meta-analysis. Ann Surg. 2008;247:49-57.

2. Madoff DC, Abdalla EK, Vauthey JN. Portal vein embolization in preparation for major hepatic resection: evolution of a new standard of care. J Vasc Interv Radiol. 2005;16:779-90.

3. Hirai I, Kimura W, Fuse A, et al. Evaluation of preoperative portal embolization for safe hepatectomy, with special reference to assessment of nonembolized lobe function with 99 mTc-GSA SPECT scintigraphy. Surgery. 2003;133:495-506.

4. Kubo S, Shiomi S, Tanaka H, et al. Evaluation of the effect of portal vein embolization on liver function by $(99 \mathrm{~m}) \mathrm{tc}$-galactosyl human serum albumin scintigraphy. J Surg Res. 2002;107:113-8.

5. Nanashima A, Yamaguchi H, Shibasaki S, et al. Relationship between CT volumetry and functional liver volume using technetium-99 m galactosyl serum albumin scintigraphy in patients undergoing preoperative portal vein embolization before major hepatectomy: a preliminary study. Dig Dis Sci. 2006;51:1190-5.

6. Uesaka K, Nimura Y, Nagino M. Changes in hepatic lobar function after right portal vein embolization. An appraisal by biliary indocyanine green excretion. Ann Surg. 1996;223:77-83.

7. Farges O, Belghiti J, Kianmanesh R, et al. Portal vein embolization before right hepatectomy: prospective clinical trial. Ann Surg. 2003;237:208-17.

8. Hemming AW, Reed AI, Howard RJ, et al. Preoperative portal vein embolization for extended hepatectomy. Ann Surg. 2003;237:686-91.

9. Aussilhou B, Lesurtel M, Sauvanet A, et al. Right portal vein ligation is as efficient as portal vein embolization to induce hypertrophy of the left liver remnant. J Gastrointest Surg. 2008;12:297-303.

10. Azoulay D, Castaing D, Krissat J, et al. Percutaneous portal vein embolization increases the feasibility and safety of major liver resection for hepatocellular carcinoma in injured liver. Ann Surg. 2000;232:665-72.

11. Azoulay D, Castaing D, Smail A, et al. Resection of nonresectable liver metastases from colorectal cancer after percutaneous portal vein embolization. Ann Surg. 2000;231:480-6.

12. Nagino M, Kamiya J, Nishio $H$, et al. Two hundred forty consecutive portal vein embolizations before extended hepatectomy for biliary cancer: surgical outcome and long-term follow-up. Ann Surg. 2006;243:364-72.
13. Ribero D, Abdalla EK, Madoff DC, et al. Portal vein embolization before major hepatectomy and its effects on regeneration, resectability and outcome. Br J Surg. 2007;94:1386-94.

14. Makuuchi M, Thai BL, Takayasu K, et al. Preoperative portal embolization to increase safety of major hepatectomy for hilar bile duct carcinoma: a preliminary report. Surgery. 1990; 107:521-7.

15. Abdalla EK, Barnett CC, Doherty D, et al. Extended hepatectomy in patients with hepatobiliary malignancies with and without preoperative portal vein embolization. Arch Surg. 2002;137: 675-80.

16. Abdalla EK, Adam R, Bilchik AJ, et al. Improving resectability of hepatic colorectal metastases: expert consensus statement. Ann Surg Oncol. 2006;13:1271-80.

17. Schindl MJ, Redhead DN, Fearon KC, et al. The value of residual liver volume as a predictor of hepatic dysfunction and infection after major liver resection. Gut. 2005;54:289-96.

18. Elias D, de BT, Roche A, et al. During liver regeneration following right portal embolization the growth rate of liver metastases is more rapid than that of the liver parenchyma. $\mathrm{Br} \mathrm{J}$ Surg. 1999;86:784-8.

19. Barbaro B, Di SC, Nuzzo G, et al. Preoperative right portal vein embolization in patients with metastatic liver disease. Metastatic liver volumes after RPVE. Acta Radiol. 2003;44:98-102.

20. Kokudo N, Tada K, Seki M, et al. Proliferative activity of intrahepatic colorectal metastases after preoperative hemihepatic portal vein embolization. Hepatology. 2001;34:267-72.

21. Heinrich S, Jochum W, Graf R, et al. Portal vein ligation and partial hepatectomy differentially influence growth of intrahepatic metastasis and liver regeneration in mice. $J$ Hepatol. 2006;45:35-42.

22. Kawasaki S, Makuuchi M, Kakazu T, et al. Resection for multiple metastatic liver tumors after portal embolization. Surgery. 1994;115:674-7.

23. Jaeck D, Oussoultzoglou E, Rosso E, et al. A two-stage hepatectomy procedure combined with portal vein embolization to achieve curative resection for initially unresectable multiple and bilobar colorectal liver metastases. Ann Surg. 2004;240:1037-49.

24. Hayashi S, Baba Y, Ueno K, et al. Acceleration of primary liver tumor growth rate in embolized hepatic lobe after portal vein embolization. Acta Radiol. 2007;48:721-7.

25. Imamura $H$, Shimada R, Kubota M, et al. Preoperative portal vein embolization: an audit of 84 patients. Hepatology. 1999;29:1099105.

26. Di Stefano DR, de BT, Denys A, et al. Preoperative percutaneous portal vein embolization: evaluation of adverse events in 188 patients. Radiology. 2005;234:625-30.

27. Alwayn IP, Verbesey JE, Kim S, et al. A critical role for matrix metalloproteinases in liver regeneration. J Surg Res. 2008;145: 192-8.

28. Mook OR, Frederiks WM, Van Noorden CJ. The role of gelatinases in colorectal cancer progression and metastasis. Biochim Biophys Acta. 2004;1705:69-89.

29. Taub R. Liver regeneration: from myth to mechanism. Nat Rev Mol Cell Biol. 2004;5:836-47.

30. Nabeshima K, Shimao Y, Inoue $T$, et al. Hepatocyte growth factor/scatter factor induces not only scattering but also cohort migration of human colorectal-adenocarcinoma cells. Int J Cancer. 1998;78:750-9.

31. Brozek W, Bises G, Girsch T, et al. Differentiation-dependent expression and mitogenic action of interleukin-6 in human colon carcinoma cells: relevance for tumour progression. Eur J Cancer. 2005;41:2347-54.

32. Otte JM, Schmitz F, Kiehne K, et al. Functional expression of HGF and its receptor in human colorectal cancer. Digestion. 2000;61:237-46. 
33. Fazekas K, Csuka O, Köves I, et al. Experimental and clinicopathologic studies on the function of the HGF receptor in human colon cancer metastasis. Clin Exp Metastasis. 2000;18:639-49.

34. Shimizu Y, Suzuki H, Nimura Y, et al. Elevated mitochondrial gene expression during rat liver regeneration after portal vein ligation. Hepatology. 1995;22:1222-9.

35. Starkel P, Horsmans Y, Sempoux C, et al. After portal branch ligation in rat, nuclear factor kappaB, interleukin-6, signal transducers and activators of transcription 3, c-fos, c-myc, and cjun are similarly induced in the ligated and nonligated lobes. Hepatology. 1999;29:1463-70.

36. Uemura T, Miyazaki M, Hirai R, et al. Different expression of positive and negative regulators of hepatocyte growth in growing and shrinking hepatic lobes after portal vein branch ligation in rats. Int J Mol Med. 2000;5:173-9.

37. Kollmar O, Corsten M, Scheuer C, et al. Portal branch ligation induces a hepatic arterial buffer response, microvascular remodeling, normoxygenation, and cell proliferation in portal blooddeprived liver tissue. Am J Physiol Gastrointest Liver Physiol. 2007;292:G1534-42.

38. Harris AL. Hypoxia-a key regulatory factor in tumour growth. Nat Rev Cancer. 2002;2:38-47.

39. Semenza GL. Hypoxia and cancer. Cancer Metastasis Rev. 2007;26:223-4.

40. Xu Y, Pasche B. TGF-beta signaling alterations and susceptibility to colorectal cancer. Hum Mol Genet. 2007;16 Spec no 1:R14-20.

41. Rocheleau B, Ethier C, Houle R, et al. Hepatic artery buffer response following left portal vein ligation: its role in liver tissue homeostasis. Am J Physiol. 1999;277:G1000-7.

42. Lautt WW. Mechanism and role of intrinsic regulation of hepatic arterial blood flow: hepatic arterial buffer response. Am J Physiol. 1985;249:G549-56.

43. Kito Y, Nagino M, Nimura Y. Doppler sonography of hepatic arterial blood flow velocity after percutaneous transhepatic portal vein embolization. AJR Am J Roentgenol. 2001;176:909-12.

44. Yokoyama Y, Wawrzyniak A, Sarmadi AM, et al. Hepatic arterial flow becomes the primary supply of sinusoids following partial portal vein ligation in rats. J Gastroenterol Hepatol. 2006;21:1567-74.

45. Mueller L, Goettsche J, Abdulgawad A, et al. Tumor growthpromoting cellular host response during liver atrophy after portal occlusion. Liver Int. 2005;25:994-1001.

46. Bajou K, Noel A, Gerard RD, et al. Absence of host plasminogen activator inhibitor 1 prevents cancer invasion and vascularization. Nat Med. 1998;4:923-8.

47. Tanaka S, Akaike T, Fang J, et al. Antiapoptotic effect of haem oxygenase- 1 induced by nitric oxide in experimental solid tumour. Br J Cancer. 2003;88:902-9.

48. Jozkowicz A, Was H, Dulak J. Heme oxygenase-1 in tumors: is it a false friend? Antioxid Redox Signal. 2007;9:2099-117.

49. Busserolles J, Megias J, Terencio MC, et al. Heme oxygenase-1 inhibits apoptosis in Caco-2 cells via activation of Akt pathway. Int J Biochem Cell Biol. 2006;38:1510-7.

50. Ciocca DR, Calderwood SK. Heat shock proteins in cancer: diagnostic, prognostic, predictive, and treatment implications. Cell Stress Chaperones. 2005;10:86-103.
51. Aoki T, Imamura H, Hasegawa K, et al. Sequential preoperative arterial and portal venous embolizations in patients with hepatocellular carcinoma. Arch Surg. 2004;139:766-74.

52. Yamakado K, Takeda K, Matsumura K, et al. Regeneration of the un-embolized liver parenchyma following portal vein embolization. J Hepatol. 1997;27:871-80.

53. Nakao N, Miura K, Takahashi H, et al. Hepatocellular carcinoma: combined hepatic, arterial, and portal venous embolization. Radiology. 1986;161:303-7.

54. Ogata S, Belghiti J, Farges O, et al. Sequential arterial and portal vein embolizations before right hepatectomy in patients with cirrhosis and hepatocellular carcinoma. Br J Surg. 2006;93: 1091-8.

55. Yamakado K, Nakatsuka A, Tanaka N, et al. Long-term followup arterial chemoembolization combined with transportal ethanol injection used to treat hepatocellular carcinoma. J Vasc Interv Radiol. 1999;10:641-7.

56. Vetelainen R, Dinant S, van VA, et al. Portal vein ligation is as effective as sequential portal vein and hepatic artery ligation in inducing contralateral liver hypertrophy in a rat model. $J$ Vasc Interv Radiol. 2006;17:1181-8.

57. Vogl TJ, Mack MG, Balzer JO, et al. Liver metastases: neoadjuvant downsizing with transarterial chemoembolization before laser-induced thermotherapy. Radiology. 2003;229:457-64.

58. Nabil M, Gruber T, Yakoub D, et al. Repetitive transarterial chemoembolization (TACE) of liver metastases from renal cell carcinoma: local control and survival results. Eur Radiol. 2008;18:1456-63.

59. Vogl TJ, Zangos S, Eichler K, et al. Colorectal liver metastases: regional chemotherapy via transarterial chemoembolization (TACE) and hepatic chemoperfusion: an update. Eur Radiol. 2007; 17:1025-34.

60. Beal IK, Anthony S, Papadopoulou A, et al. Portal vein embolisation prior to hepatic resection for colorectal liver metastases and the effects of periprocedure chemotherapy. $\mathrm{Br} J$ Radiol. 2006;79:473-8.

61. Goere D, Farges O, Leporrier J, et al. Chemotherapy does not impair hypertrophy of the left liver after right portal vein obstruction. J Gastrointest Surg. 2006;10:365-70.

62. Covey AM, Brown KT, Jarnagin WR, et al. Combined portal vein embolization and neoadjuvant chemotherapy as a treatment strategy for resectable hepatic colorectal metastases. Ann Surg. 2008;247:451-5.

63. Selzner N, Pestalozzi BC, Kadry Z, et al. Downstaging colorectal liver metastases by concomitant unilateral portal vein ligation and selective intra-arterial chemotherapy. Br J Surg. 2006;93:587-92.

64. Abdalla EK, Hicks ME, Vauthey JN. Portal vein embolization: rationale, technique and future prospects. Br J Surg. 2001;88: 165-75.

65. Nishiyama Y, Yamamoto Y, Hino I, et al. 99mTc galactosyl human serum albumin liver dynamic SPET for pre-operative assessment of hepatectomy in relation to percutaneous transhepatic portal embolization. Nucl Med Commun. 2003;24:809-17.

66. Benoist S, Nordlinger B. Neoadjuvant treatment before resection of liver metastases. Eur J Surg Oncol. 2007;33(Suppl 2): S35-41. 